\title{
MARCA CUIDAD PARA SANTIAGO DE CALI MEDIANTE LA METODOLOGÍA BÁSICA DE DISEÑO
}

\section{BRAND CITY FOR SANTIAGO DE CALI THROUGH THE BASIC DESIGN METHODOLOGY}

\author{
María elvira Arboleda Castro, Camilo Velasco Cano y Sebastián Zuluaga Piedrahíta
}

Grupo de investigación economía, políticas públicas y métodos cuantitativos

Universidad Icesi

Recibido: 13/03/2021 Aprobado: 24/05/2021

Cómo citar este artículo:

Arboleda Castro, M.E., Velasco Cano, C. y Zuluaga Piedrahíta, S. (2021). Marca ciudad para Santiago de Cali mediante la metodología básica de diseño. Revista Sapientía, 13(26), 8-16.

\section{RESUMEN}

"Cali, lo mejor de la gente" es una marca diseñada para la ciudad Santiago de Cali con el fin de aportar a la construcción de las relaciones entre los ciudadanos y el civismo de la ciudad. Creada mediante la metodología básica de diseño que busca satisfacer las necesidades de la audiencia, la imagen de marca sintetiza la ciudad y la imagen se convierte en un punto de referencia que puede llegar a ser una fuente de inspiración para mejorar el comportamiento individual y colectivo de sus ciudadanos. Esto con el fin de posicionar una ciudad y su producción en los distintos mercados y, desde una imagen, movilizar al ciudadano por una causa común: el territorio común, la ciudad como espacio apto para producir calidad de vida.

El diseño de una marca ciudad puede coadyuvar a trazar el rumbo que tendrá la ciudad de Santiago de Cali y sus habitantes en conjunto con los gobiernos locales. Es así como la implementación de políticas gubernamentales que contribuyan al desarrollo de la identidad local permitirá que la ciudad tenga un direccionamiento claro y establecerá nuevas metas para la urbe y sus habitantes.

Esta investigación busca elaborar una metodología básica de diseño para crear la marca de ciudad y planear un modelo de ciudad idóneo.

\section{PALABRAS CLAVE}

Marketing, marca ciudad, metodología básica de diseño, Santiago de Cali.

\section{ABSTRACT}

"Cali, the best of the people" is a brand designed for the city of Santiago de Cali, in order to contribute to the construction of relationships between citizens and the civic spirit of the city. Created through the basic design methodology, which seeks to satisfy the needs of the audience, the brand image synthesizes the city and the image becomes a point of reference, which can become a source of inspiration to improve individual behavior and collective of its citizens. This in order to position a city and its production in the different markets and, from an image, mobilize the citizen for a common cause, the common territory, the city as a space suitable to produce quality of life.

The design of a city brand can help to chart the direction that the city of Santiago de Cali will take, and its inhabitants in conjunction with local governments. This is how the implementation of government policies that contribute to the development of local identity, will allow the city to have a clear direction and will establish new goals for the city and its inhabitants.

This research seeks to develop a basic design methodology, to create the city brand, and plan an ideal city model. 


\section{KEYWORDS}

Marketing, city brand, basic design methodology, Santiago de Cali.

\section{INTRODUCCIÓN}

Durante los años 90, las modalidades de gestión urbana se transformaron simultáneamente con el desarrollo de las grandes ciudades. En ese entonces solo se tenía un nombre como tal, una bandera con escudo y un himno que la identificara, ahora, en el curso del siglo XXI, se cuenta con una marca con logotipo, una imagen corporativa, un slogan y un mercadeo propio que las identifica como ciudad.

Uno de los principales objetivos es crear un modelo de ciudad para trazar su visión de futuro. Los diversos agentes que manejan una ciudad gestionan y toman decisiones para definir y alcanzar un modelo de ciudad con un propósito estratégico y con metas claras.

Por esta razón, una marca ciudad para Santiago de Cali es un elemento importante para la construcción cultural y social de la ciudad. La presente investigación es el reflejo de un proceso enfocado en el diseño centrado en el ser humano, mediante la construcción de un elemento visual y conceptual para la solución puntual de un problema cívico encontrado en la ciudad. El problema está planteado en un reto que da lugar a una metodología diseñada por Básica, una empresa de diseño de la ciudad de Medellín que, tomando en cuenta diferentes conceptos de diseño, crea un proceso único y acertado para darle solución a cualquier tipo de reto que incluya a una audiencia dentro de la solución: la metodología Básica de diseño.

Como lo señalan Calvento \& Colombo (2009), la marca ciudad puede concebirse como el "nombre, término, símbolo o diseño, o combinación de ellos que trata de identificar las características de la ciudad y así mismo diferenciarla de otras ciudades". Una marca ciudad se define de manera apropiada como la manera de implementar las estrategias de mercadeo de las ciudades. Siguiendo a los autores, la marca ciudad es un distintivo que permite destacar las fortalezas de la ciudad permitiendo llegar y atraer a un mayor público, así logra ser un atractivo logrando ser más competitiva.

El objetivo de la marca-ciudad, como lo argumenta Calvento \& Colombo, (2009), es:

Potenciar las capacidades de los territorios, desplegar sus ventajas competitivas y posicionarse nacional e internacionalmente, entre otros objetivos de esta estrategia de marca ciudad se destaca el fortalecimiento de las relaciones entre los ciudadanos para hacer más atractiva su estadía y desarrollo en la ciudad. Como se menciona dentro de la investigación, la aplicación del marketing de ciudad depende en gran medida de la construcción, comunicación y gestión de la imagen de la ciudad, ya que esta se crea a través de percepciones e imágenes.

En tal sentido, se hace necesario crear una metodología básica de diseño de marca para la ciudad de Santiago de Cali, donde esta se verá como un producto significativo para que la economía se constituya con una visión multidisciplinaria dentro de un mercado y que las marcas y los consumidores de ellas se vean inmersas en un solo contexto, es decir, que la marca ciudad sea la identidad multicultural basada en la alegría de su gente, su cultura, etc., la cual pude ir más allá incluyendo una visión, como lo son las nuevas metas y nuevas estrategias.

Bajo este concepto lo que se pretende es buscar iniciativas que sirvan como herramienta para buscar la ciudad que se quiere, en las cuales se puedan conjugar las fortalezas que encaminan al fortalecimiento de las debilidades. Una de estas opciones es buscar en los objetivos la creación de un concepto-imagen que identifique la urbe. La investigación se centró en la ciudad Santiago de Cali, en su cultura, sus habitantes, su alegría, sus tradiciones y sus lugares más importantes; teniendo en cuenta variables 
determinantes como: nivel de educación de sus habitantes, estrato socio económico, origen, etnia, religión, ocupación, estado civil, edad y actividades como deportes o pasatiempos a las que se encuentran vinculados sus habitantes.

La investigación resulta de gran interés por cuanto deja en evidencia que el papel más activo que tiene el ciudadano en la actualidad al intervenir en las decisiones que afectan a la ciudad, involucrándose e interesándose por aspectos como los valores que conforman su ciudad. Al respecto, Lablanca (2014) señala que las nuevas dinámicas que están transformando la ciudad empiezan por unos ciudadanos exigentes ante el servicio ofrecido por los administradores públicos encargados de gestionar la ciudad, los cuales dejan de ser administrados para convertirse en clientes. Para la autora, la colaboración público-privada es un elemento clave para lograr una identidad de marca adecuada que genere entusiasmo por la marca y que el ciudadano sienta apego por ella.

\section{MARCO TEÓRICO}

El termino marketing empezó a utilizarse en Estados Unidos a principios del siglo XX, concretamente en el año 1902. El marketing consiste en identificar y satisfacer las necesidades de las personas y de la sociedad (Keller, 2006, p.5). Al mismo tiempo, la American Marketing Association (2016) (Asociación Americana de Marketing) define el termino marketing como "el conjunto de procesos para generar, comunicar y entregar valor a los consumidores”. En otras palabras, se entiende el término marketing como la manera de satisfacer necesidades de manera rentable. Además, el Marketing es una manera de pensar, una filosofía de dirección, sobre cómo debe entenderse la relación de intercambio de los productos/servicios de una organización en el mercado (Vásquez, 2000). Entre las diferentes conceptualizaciones se puede distinguir la definición social y la definición empresarial. La definición social refleja la función que debe desempeñar el marketing en la sociedad, por lo tanto, la función del marketing es lograr un mejor nivel de vida en los consumidores. En cuanto a la definición empresarial, el marketing se ha descrito a menudo por muchos autores como el arte de vender productos.

Como señalan Calvento \& Colombo (2009), la marca ciudad "debe convertirse en un activo altamente estratégico que transmita efectivamente la esencia y la identidad del territorio para potenciar los valores culturales y la imagen de la ciudad". En tal sentido, los autores en mención enfatizan en que toda ciudad tiene una imagen, pero lo más importante es evolucionar hacia algo más poderoso, que agregue valor a los esfuerzos públicos, institucionales y comerciales, en general a todas las actividades donde se involucra la identidad de la ciudad. Es así como la marca-ciudad permite definir una serie de atributos singulares y exclusivos por los que se puede identificar, reconocer y diferenciar una ciudad de otras.

Sumado a lo anterior, con relación a la estrategia de creación y desarrollo de una marca ciudad, como se menciona en la investigación "La ciudad como producto", Lablanca (2014) plantea que es importante promover la participación de los residentes, ya que la creación de toda marca debe darse a partir de lo que ve el ciudadano y de cómo lo interpreta con base en su sentido de pertenencia. Los ciudadanos al ser involucrados en el proceso generarán una buena voluntad para promocionar y mejorar la ciudad, logrando una mejoría en la calidad de sus servicios. En la misma investigación, la creación de la marca ciudad se desprende de las personas y los valores que habitan en ellas, valores conformados por la cultura, la historia, la personalidad y los gustos. Lablanca (2014), con base en los planteamientos de Toni Puig, especialista en marketing de ciudad, afirma que la creación de la marca ciudad se desarrolla a partir de la manera en que la ciudad toma forma en la mente de las personas, es decir, como las personas entienden la ciudad a partir de sus propias percepciones. Por esta razón, para el autor la creación de la marca ciudad se debe pensar a partir de los estados de opinión de los ciudadanos, ya que estos han adquirido un papel fundamental en cada una de las decisiones donde se involucra la ciudad. 
Considerando estos planteamientos, la participación ciudadana es de gran importancia en el desarrollo de la marca ciudad, por lo que esta tendrá que estar asociada a un grupo de atributos que serán percibidos por el público objetivo positivos, está series de atributos tendrá que estar relacionada con el nombre de la marca ciudad, el logo y el eslogan.

A juicio de Lablanca (2014),"El desafío de la construcción de la marca ciudad está en crear una serie de profundas asociaciones positivas que conecten con las percepciones y pensamientos del público objetivo".

Govers, junto con Van't Klooster y Van Keken (2015) de los países bajos desarrollaron un conjunto de principios de marca lugar para guiar el desarrollo y gestión de marca de ciudades, regiones, destinos y países. Para estos autores el place branding puede ayudar construyendo una identidad competitiva que influya en las imágenes percibidas por los mercados más relevantes. El place branding, según expresan los autores, consiste básicamente en tender puentes entre la identidad o carácter de un lugar, su oferta de producto, la experiencia que obtienen los visitantes (turistas, expatriados, inversores potenciales $\mathrm{o}$ comerciantes) y la imagen o percepción que la gente tiene de ese territorio.

Para Govers, Van't Klooster y Van Keken (2015) la comunicación es una de las áreas más estratégicas e importantes en el desarrollo y gestión de la marca ciudad, pues es aquella herramienta estratégica que ayudará a la difusión de la misma una vez creada para determinar el reconocimiento y posteriormente el éxito del territorio, pero probablemente resulta más importante aún en el place branding la construcción y la cooperación, conceptos a desarrollar.

\section{Según Browm (2008).}

La construcción y la gestión de la marca ciudad consisten en el desarrollo de productos o acciones estratégicas que apoyen a las promesas de la marca como, por ejemplo, desarrollo de infraestructuras, eventos, programas para mejorar de la calidad cooperativa, así como la creación de iconos u otras acciones simbólicas. Por su parte, la cooperación consiste encontrar puntos de acuerdo y objetivos comunes entre las partes interesadas, el sector público, privado y la sociedad civil, con el objetivo de determinar qué es lo que hace a un lugar en único y atractivo, y de asegurar que las instituciones, las empresas y los ciudadanos también "viven de la marca".

\section{ESTRATEGIAS PARA LA CONSTRUCCIÓN DE UNA MARCA CIUDAD}

Desde el punto de vista de Sáez (2011).

La marca ciudad ha de comprender aspectos importantes como la definición de una imagen fuerte, diferente, coherente y sobre todo creadora de una imagen favorable en el público objetivo. De esta manera la autora define la base para la creación de una marca ciudad efectiva por medio de los aspectos mencionados que tienen como objetivo diferenciar a la ciudad en el territorio nacional y garantizar una fuerza competitiva que la posicione de manera local e internacional.

Así mismo, como indica Lablanca (2014), la ciudad debe constar de algunas características propias que son un instrumento o una manera de competir para diferenciarse de otras ciudades. En este contexto, una de las características más importantes de cualquier ciudad que le permite diferenciarse de otras ciudades es la "imagen" y junto con esta su "marca e imagen de marca", teniendo en cuenta estos dos aspectos, es posible percibir el alto valor que conlleva la creación de una marca ciudad como estrategia competitiva y diferenciadora de otras ciudades. Para Lablanca (2014), la marca como activo importante de la ciudad no deberá dejarse al azar y debe ser contemplado dentro del plan estratégico de cada gobierno.

\section{METODOLOGÍA BÁSICA DE DISEÑO}

Hace 13 años, Juan Camilo Vélez, ingeniero industrial de la Escuela de Ingeniería de Antioquia; Paulo Carvajal, ingeniero y administrador de la Universidad Nacional de Colombia, y Jaime Villa, administrador de empresas y mercadólogo de la Universidad CEIPA de Medellín, decidieron crear una agencia de publicidad con un alto grado de diferenciación. Después de haber trabajado en múltiples empresas y dedicado su vida a la publicidad y el mercadeo se dieron cuenta de la necesidad de cambiar el rumbo de la publicidad. Enfocaron sus fuerzas en el estudio de una manera diferente de generar comunicación y hacer del mercadeo y la publicidad una práctica más humana.El inicio de esta búsqueda se remonta a la Universidad de Stanford, donde hace aproximadamente más de 10 años esta institución desarrolla una investigación que da cuenta del poco nivel de humanismo que contienen 


\section{2 | Sapıentia Vol. 13, №. 26, noviembre 202l. pág. 8-16 ISSN I909-08II}

las grandes empresas del mundo. Una de las razones es que los presidentes y vicepresidentes de las grandes compañías tenían como objetivo extraer la mayor cantidad de valor para los inversionistas. La Universidad de Stanford comienza a ejecutar un plan para cambiar esta realidad. En ese momento es donde se dan cuenta de la existencia de un instituto de diseño creado después de la Segunda Guerra Mundial, llamado Bauhaus. Este centraba todos sus esfuerzos en cómo resolver la vida del humano.

A partir de esto, los fundadores de Básica deciden encontrar la manera de crear una metodología de diseño enfocada en el humano. Por esta razón, Jaime Villa, gerente actual de la agencia, comienza a estudiar diferentes teorías y metodologías ya existentes que pudiesen funcionar para crear la Metodología Básica de Diseño. En primer lugar, comienzan a estudiar y a entender el término Design Thinking, el cual fue creado en las aulas de la Universidad de Stanford y popularizado por Tim Brown y David Kalley. Esta teoría, aunque se explica a profundidad más adelante, propone "un método que nos permite generar soluciones innovadoras basadas en las necesidades de los usuarios". Sin embargo, para Jaime no era suficiente basar su metodología sólo en este concepto, por lo que decide estudiar otras teorías y herramientas que le agregaran valor a su metodología. En primer lugar, decide estudiar la propuesta de valor modelo Canvas, concepto creado por Osterwalder (2015) y Pigneur (2015), expuesto en su libro Business Model Generation. Este concepto le da visión de cómo desarrollar modelos de negocios rentables, que aporten valor a los humanos mediante productos o servicios innovadores. Luego, realiza un acercamiento a la metodología To Be Done (2019), creada por Christensen (2019), la cual ayuda a entender que las marcas deben enfocarse en lo que el público quiere resolver en su vida y no lo que quiere comprar.

\section{¿EN QUÉ CONSISTE LA METODOLOGÍA BÁSICA DE DISEÑO?}

Siendo liberar lo sagrado del humano el MTP o Propósito Masivo de Transformación de la agencia Básica, sus socios deciden estructurar una metodología que cumpliera con la creación de marcas, estrategias, propuestas y empresas que pudiesen cum- plir con el objetivo de cambiar el mundo. Por lo cual deciden incluir los conceptos anteriormente mencionados en las diferentes etapas de la metodología.

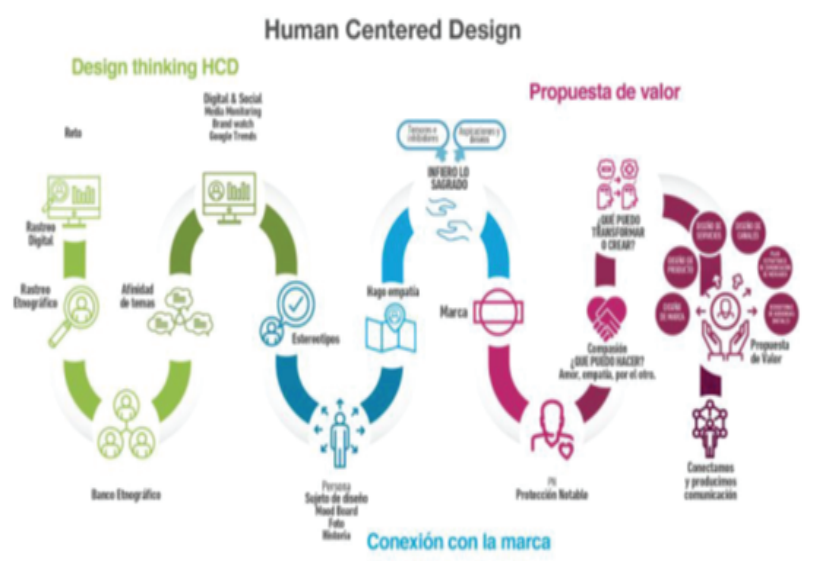

Fig. 1. Human Centred Design Fuente: Básica S.A.S (2012)

Básica como agencia propone a sus clientes el desarrollo de esta metodología en el orden expuesto en la Figura 1, con el fin de conectar y generar comunicación para sus marcas. Esta práctica se divide en varias etapas, cada una de ellas con un objetivo específico:

\section{ETAPAS DE LA METODOLOGÍA BÁSICA DE DISEÑO}

\section{El reto}

El reto es el trigger de la metodología, es decir, es el disparador para todo lo que va suceder a lo largo del proceso. La idea de Básica en este punto es hacer que las marcas planteen un reto que debe quedar escrito con un cómo y un para, esto con el fin de entender que necesita solucionar la marca o a dónde quiere llegar con algún tema en específico. El planteamiento de la pregunta reto cuenta con dos componentes claves, el dolor y la solución. El dolor es esa solución al problema, el cual es representado en la pregunta con el cómo. La solución es, en este caso, el objetivo al cual se quiere llegar, el cual está representado en la pregunta con el para.

Es así como la investigación explica la importancia de adaptar estrategias del branding aplicadas a la ciudad para generar percepciones positivas por parte del públ- 
ico potencial en relación con el desarrollo y proyección de una imagen determinada. Como menciona Lablanca (2014), la marca ciudad ha de contemplar la definición de una imagen fuerte, diferente, reconocible, coherente y sobre todo creadora de una opinión favorable entre los públicos a los que se dirige. De tal forma, las estrategias de branding serán la base para la creación de una marca ciudad efectiva y diferenciadora, que además garantice una fuerza competitiva que permita a la ciudad posicionarse en la mente de distintos públicos objetivo. La investigación aborda como principios del branding aplicados a la marca ciudad la identificación, la diferenciación, el posicionamiento y la segmentación del público objetivo.

\section{METODOLOGÍA DE LA INVESTIGACIÓN}

En una sesión de dos horas se establecieron unos parámetros teniendo en cuenta el contexto de lo que se quiere resolver con esta metodología. Mediante una discusión de la situación actual de la ciudad y haciendo una revisión adelantada, pero superficial de los hallazgos encontrados en la segunda parte de esta metodología. Se comenzaron a entregar ideas de lo que podría ser el reto por resolver. A continuación, se mostrarán las opciones anotadas en la sesión:

- Opción 1: ¿Cómo generar civismo en Cali para entregarles una mejor calidad de vida a sus ciudadanos?

- Opción $2:$ ¿Cómo cambiar la mentalidad de los caleños para hacer de Cali una ciudad más tranquila y con una mejor calidad de vida?

- Opción $3:$ ¿Cómo enamorar a los caleños de su ciudad mediante una marca para lograr un mejor comportamiento en sus ciudadanos?

- Opción $4:$ ¿Cómo entregar una marca con un mensaje fuerte y claro para mejorar el civismo en Cali?
- Opción $5:$ ¿Cómo crear una marca que enamore a los caleños para que cuiden más de su ciudad y su gente?

Opción 6 : ¿Cómo enamorar a los caleños de su ciudad y su gente mediante una marca, para aportar a la construcción del civismo?

Opción 7: ¿Cómo crear una marca empática para mejorar el civismo en Cali?

Las anteriores son las opciones que salieron a relucir dentro de la discusión del reto. El paso a seguir fue hacer un análisis de las mismas y escoger la que más se acercaba a lo que se puede lograr de manera realista y clara con esta metodología. Al final, la opción escogida fue la número 6 (¿Cómo enamorar a los caleños de su ciudad y su gente mediante una marca, para aportar a la construcción del civismo?), dado que era la pregunta que más se acercaba a lo que se podría lograr dado que recoge tres componentes importantes. El primero es la palabra enamorar, pues es un reto interesante que guía el desarrollo de la marca por el mundo de la empatía y el sentimiento. El segundo es la inclusión de la ciudad y su gente como objetivo, pues en el contexto de la investigación de la audiencia, quedó claro que para el caleño es muy importante su gente y sus compañeros de ciudad, y no solamente su ciudad como tal, y finalmente el componente del civismo, el cual es un reto muy grande por el cual todas las ciudades del mundo trabajan. En esta parte de la pregunta, el análisis realizado consiste en que la pregunta no apela a resolver del todo el problema del civismo en la ciudad, sino que está planteada como un sistema de apoyo a la construcción del mismo aportando a él mas no resolviéndolo del todo, lo cual se acerca a la realidad más que en las otras opciones. Así, el reto escogido da paso a seguir desarrollando la metodología con una pregunta que será la encargada de guiar el camino y enfocar los esfuerzos en ella. 


\section{RASTREO DIGITAL}

Dentro de este proceso la investigación realizada se basó en estudiar las redes sociales y observarlas con el fin de encontrar diferentes afirmaciones, publicaciones, noticias, opiniones, etc., que dieran un conocimiento específico de la audiencia. Una de las redes que más se estudió fue Twitter, donde se encontraron diferentes opiniones y noticias que aportaron a la construcción de la información para definir la audiencia y entenderla. Este proceso también fue aplicado en redes como Instagram, donde se estudiaron los perfiles de algunos caleños con el fin de encontrar comportamientos, gustos y opiniones sobre sus experiencias en la ciudad. En este caso, la información fue mucho más precisa y ayudó al entendimiento de los estereotipos de personas que podemos encontrar entre los ciudadanos de Cali. En promedio se observaron cincuenta perfiles de personas de diferentes estratos y con diferentes contextos.

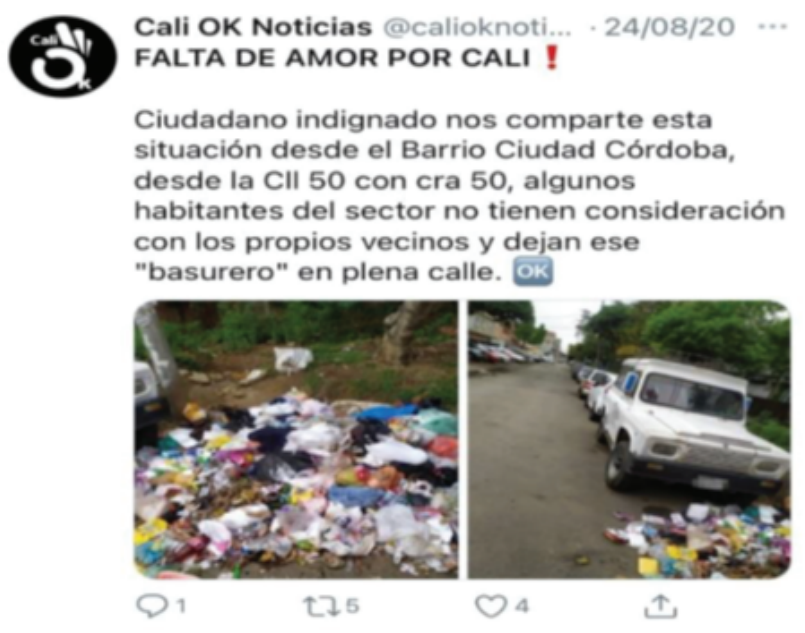

Fig. 2. Percepción de los ciudadanos caleños Fuente: Elaboración propia

La siguiente lista hace referencia a los temas o frases expresados por la audiencia en relación a la ciudad de Cali. Estos temas fueron evidenciados en el rastreo digital realizado. Como la metodología lo propone, se tomó la tarea de evaluar cuáles fueron los temas más relevantes o repetidos por la audiencia. A partir de esto se comenzó a planear un escenario donde los temas más relevantes fueron conformando los problemas o dolores y las ventajas o valores más expuestos en los ciudadanos caleños. Para tener mayor claridad del tema, a continuación se listan los 5 temas más relevantes encontrados en la audiencia:

1. La amabilidad de los caleños.

2. Falta de civismo

3. Sentido individualista entre los caleños.

4. Energía única de los caleños.

5. El caleño siempre lo disfruta todo.

\begin{tabular}{|l|l|l|}
\hline La inseguridad en Cali & Ciudad musical. & Ambiente de celebración \\
\hline El sabor de los caleños. & Individualidad del caleño. & Lugares icónicos. \\
\hline La amabilidad de los caleños. & Clima perfecto. & Deseo de cultura paisa. \\
\hline La numba de Cali. & Contaminación & Pocas oportunidad laboral. \\
\hline Egoismo entre los caleños. & Cali es alegria & Gobiemo comupto. \\
\hline Eventos culturales importantes. & Energia única del caleño. & Falta de amor por Cali. \\
\hline
\end{tabular}

Tabla 1. Temas o frases expresados por la audiencia con la relación a la ciudad de Cali

Fuente: elaboración propia

La protección notable es, en otras palabras, lo que la marca va a aportar al sujeto de diseño, lo que le va a ayudar a aspirar o alcanzar. En esta metodología la marca se hace a un poder con el cual puede agregarle valor a su audiencia apelando a los Jobs to be done, explicados en el marco teórico. Es decir, a lo que el sujeto realmente necesita que se le sea solucionado o protegido. En este caso fue escogida una aspiración propuesta en el mapa de empatía para Velería, la cual es "que todos los caleños amen la ciudad como ella". Esta aspiración va a estar apalancada por el poder de la marca, en este caso la ciudad de Cali, la cual tiene como poder 'ser lo mejor de la gente'”. La protección notable se resume en la siguiente figura: 


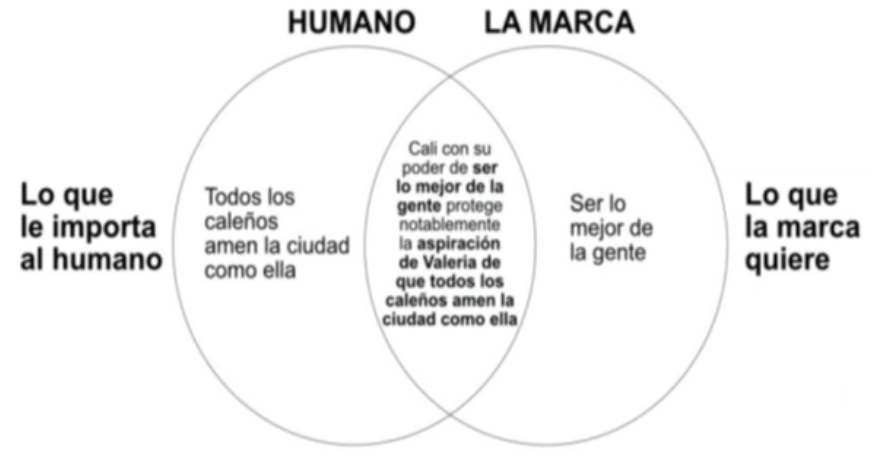

Fig. 3. El poder de la marca Fuente: elaboración propia.

\section{DESARROLLO DE MARCA}

Es este punto se ve el resultado de la metodología, la cual permitió encontrar insights importantes sobre los componentes más importantes de la ciudad de Cali. De estos insights, uno que generaba mayor empatía con el sujeto de diseño $y$ fue mencionado notoriamente en las entrevistas a profundidad y en el rastreo desarrollado en el proceso de conocimiento de audiencia. Este insight hace referencia a la gente, en primer lugar, se encontró una diferenciación en las cualidades de los habitantes de Cali. Una ciudad que se caracteriza por su calidez humana, por personas con gran corazón y con la disponibilidad de ayudar a la comunidad cuando lo necesite; este rasgo sería uno de los elementos por los cuales los habitantes de la ciudad de Cali son recordados y extrañados con frecuencia cuando se está lejos de Cali. Dicho hallazgo fue repetitivo en las entrevistas a profundidad y nos hace pensar que la calidez humana de nuestra ciudad nos hace mucho más confortables, empáticos y diferenciadores.

\section{CONCLUSIONES}

En primer lugar, en el desarrollo de la investigación de la audiencia se evidenció que la ciudad de Santiago de Cali tiene un mal posicionamiento a nivel regional en cuanto a la seguridad se refiere. Las personas que participaron de las entrevistas a profundidad y los hal- lazgos encontrados en el rastreo manifestaron esta inconformidad en repetidas ocasiones. Sin embargo, en la investigación no todo fue de carácter negativo, de hecho, uno de los temas más representativos dentro del proceso fue el manifiesto de las personas sobre la amabilidad de las personas de Cali y su alegría constante, dejando claro que los caleños son personas de calidad.

A pesar de esto, manifestaron una inconformidad constante sobre el comportamiento de los ciudadanos en situaciones particulares, que en su mayoría refiere a temas de civismo, afirmando la falta de este en los ciudadanos. La inclusión del concepto de la marca sobre la gente fue respaldado por lo dicho anteriormente, ya que fue lo que más se resaltó y, así mismo, apelando a dar a conocer esta diferenciación con el fin de mejorar el civismo en la ciudad. Por otro lado, es fácil concluir que la metodología dejó en evidencia estos conceptos de manera muy clara y que fue una herramienta fundamental para desarrollar el trabajo y encontrar los problemas y necesidades de los ciudadanos. La metodología fue precisa, permitió conocer y entender al ciudadano caleño de manera completa, llevando el trabajo a un excelente resultado de concepto y marca.

Finalmente, en el desarrollo del trabajo se encontró que la estrategia de marca ciudad tiene como objetivo reconocer las ventajas competitivas de un territorio y potenciarlas a nivel nacional para generar un buen posicionamiento de la ciudad, permitiendo que esta sea atractiva para turistas, ciudadanos, empresas e inversionistas. Por esta razón, es de gran importancia que las ciudades cuenten con esta estrategia dentro de sus planes regionales para el desarrollo económico y cultural. Con la estrategia "Cali, lo mejor de la gente" se abarcó todo lo dicho anteriormente; con esta marca se logra transmitir una muy buena imagen de la ciudad para el exterior y además dar un mensaje fuerte y contundente a los ciudadanos de Cali para que comprendan que Cali puede progresar dependiendo de un solo factor, su gente. 


\section{RECOMENDACIONES}

En próximas investigaciones se deberá identificar los elementos que logran unificar la cultura caleña. Cali, a pesar de ser una ciudad multicultural, cuenta con el ritmo que sus habitantes se han encargado de construir a través del tiempo, elemento que no solo se usa para el baile, sino también para resaltar que los caleños mantienen el ritmo en cada una de sus actividades.

\section{REFERENCIAS BIBLIOGRÁFICAS}

Calvento, M. y Colombo, S. S. (2009). La marca ciudad como herramienta de promoción turística: ¿Instrumento de inserción nacional e internacional? Estudios y Perspectivas en Turismo, 18 (3), 262-284. https://www. redalyc.org/articulo.oa?id=180714243002

Kotler, P. y Keller, K.L. (2006). Dirección de Pearson.

Lablanca, M. (2014). La ciudad como producto. Aproximación a las estrategias de marketing del sector empresarial aplicadas en la gestión de la ciudad. La marca ciudad. El modelo de construcción de la marca ciudad en Sant Cugat (Trabajo de grado no publicado). Universidad Autónoma de Barcelona, España.

Sáez, L., Mediano, L., Elizagárate, V. (2011). Creación y desarrollo de marca ciudad. Análisis de los registros de marca de las principales ciudades españolas. Revista de Dirección y Administración de Empresas, 18, 125- 156.

Vásquez, M. A. (2000). Marketing social corporativo. Juan Carlos Martínez Coll.

\section{AUTORES}

María Elvira Arboleda Castro: docente investigadora de la Universidad Autónoma de Occidente, Institución Universitaria Antonio José Camacho, Universidad Icesi y Universidad de San Buenaventura Cali. Economista, Magíster en Economía y candidata a Doctora en Economía e Historia Económica de la Universidad de Sevilla, España. Correo: marearboleda2011@hotmail.com

Camilo Velasco Cano: estudiante de Mercadeo Internacional y Publicidad de la Universidad Icesi. Correo: camilo.velasco@outlook.com

Sebastián Zuluaga Piedrahíta: Estudiante de Mercadeo Internacional y Publicidad Universidad Icesi. Correo: 23sebastianzuluaga@gmail.com 